\title{
Hepatic Steatosis Is Associated with Increased Disease Severity and Liver Injury in Coronavirus Disease-19
}

\author{
Vincent L. Chen ${ }^{1} \odot$ - Fadi Hawa ${ }^{2}$ - Jeffrey A. Berinstein ${ }^{1}$. Chanakyaram A. Reddy ${ }^{1} \cdot$ Ihab Kassab $^{3} \cdot$ Kevin D. Platt $^{1}$. \\ Chia-Yang Hsu ${ }^{1}$. Calen A. Steiner ${ }^{1}$. Jeremy Louissaint ${ }^{1} \cdot$ Naresh T. Gunaratnam ${ }^{4}$. Pratima Sharma ${ }^{1}$
}

Received: 7 August 2020 / Accepted: 14 September 2020 / Published online: 27 September 2020

(c) Springer Science+Business Media, LLC, part of Springer Nature 2020

\begin{abstract}
Background Coronavirus disease-2019 (COVID-19) is a global pandemic. Obesity has been associated with increased disease severity in COVID-19, and obesity is strongly associated with hepatic steatosis (HS). However, how HS alters the natural history of COVID-19 is not well characterized, especially in Western populations.

Aims To characterize the impact of HS on disease severity and liver injury in COVID-19.

Methods We examined the association between HS and disease severity in a single-center cohort study of hospitalized COVID-19 patients at Michigan Medicine. HS was defined by either hepatic steatosis index $>36$ (for Asians) or $>39$ (for nonAsians) or liver imaging demonstrating steatosis $>30$ days before onset of COVID-19. The primary predictor was HS. The primary outcomes were severity of cardiopulmonary disease, transaminitis, jaundice, and portal hypertensive complications. Results In a cohort of 342 patients, metabolic disease was highly prevalent including nearly $90 \%$ overweight. HS was associated with increased transaminitis and need for intubation, dialysis, and vasopressors. There was no association between HS and jaundice or portal hypertensive complications. In a sensitivity analysis including only patients with liver imaging $>30$ days before onset of COVID-19, imaging evidence of hepatic steatosis remained associated with disease severity and risk of transaminitis.

Conclusions HS was associated with increased disease severity and transaminitis in COVID-19. HS may be relevant in predicting risk of complications related to COVID-19.
\end{abstract}

Keywords SARS-CoV-2 Acute liver injury $\cdot$ Outcomes $\cdot$ NAFLD

\section{Abbreviations}

ALT

AST

BMI

COVID-19 Coronavirus disease-2019

Vincent L. Chen

vichen@med.umich.edu

Pratima Sharma

pratimas@med.umich.edu

1 Division of Gastroenterology and Hepatology, Department of Internal Medicine, University of Michigan, $1500 \mathrm{E}$. Medical Center Dr., Ann Arbor, MI 48109, USA

2 Department of Medicine, St. Joseph Mercy Ann Arbor Hospital, Ypsilanti, MI, USA

3 Division of Hospital Medicine, Department of Internal Medicine, University of Michigan, Ann Arbor, MI, USA

4 Huron Gastro Center for Digestive Disease, Ypsilanti, MI, USA
HS Hepatic steatosis

HSI Hepatic steatosis index

IQR Interquartile range

ULN Upper limit of normal

WHO World Health Organization

\section{Introduction}

Coronavirus disease-2019 (COVID-19) is a pandemic responsible for $>25$ million cases and $>800,000$ deaths worldwide [1]. COVID-19 frequently results in gastrointestinal disease including liver injury [2, 3]. However, whether underlying liver disease influences COVID-19-related disease severity - both severity of respiratory disease and clinically meaningful liver-related outcomes such as jaundice, ascites, and hepatic encephalopathy_is less clear [4]. This question is critical because metabolic dysfunction-associated fatty liver disease, characterized by hepatic steatosis (HS) 
and metabolic risk factors including overweight, affects up to $30 \%$ of the population [5-8]. Further, metabolic risk factors are strongly associated with increased COVID-19 disease severity $[9,10]$. However, the impact of HS on COVID19-related outcomes is not well understood and has not been reported to our knowledge in the USA [11]. We aimed to evaluate the impact of HS on disease severity and liver injury in COVID-19.

\section{Methods}

This was a retrospective single-center cohort study. Our study included hospitalized adult patients ( $\geq 18$ years old) at Michigan Medicine with COVID-19 diagnosed by polymerase chain reaction between March 10, 2020 (first identified case in the state of Michigan) and May 20, 2020. Patients were followed until death, discharge from the hospital, or September 3, 2020. This study was approved by the University of Michigan Institutional Review Board.

Outcomes were disease severity and liver injury. Disease severity was defined as: death, need for intensive care unit (ICU) admission, intubation, vasopressor requirement, new dialysis requirement, or the World Health Organization (WHO) ordinal scale. Liver injury was defined as peak alanine aminotransferase (ALT) $>2 \mathrm{X}$ or $5 \mathrm{X}$ the upper limit of normal (ULN) and $>2 \mathrm{X}$ or $5 \mathrm{X}$ the patient's prior baseline ALT (if known); jaundice (total bilirubin $>2 \mathrm{mg} / \mathrm{dL}$ or $4 \mathrm{mg} /$ $\mathrm{dL}$ ); or new/worsening ascites or encephalopathy. ALT ULN was defined as $19 \mathrm{U} / \mathrm{L}$ for women and $30 \mathrm{U} / \mathrm{L}$ for men [12, 13].

The primary predictor was HS, defined by either imaging evidence of steatosis $>30$ days before COVID-19 diagnosis, or hepatic steatosis index (HSI) $>36$ for Asians and $>39$ for non-Asians [14], based on ambulatory laboratory values $>30$ days before COVID-19 diagnosis. HSI was defined as $8 *$ ALT/aspartate aminotransferase (AST) + body mass index (BMI) +2 (if diabetes) +2 (if female).

Continuous variables were represented as median (interquartile range [IQR]) and categorical variables as percentages. Continuous variables were compared using rank-sum tests and categorical variables using $\chi^{2}$ tests. Statistical significance was defined as two-tailed $P$ value $<0.05$. We used logistic regressions with disease severity/liver injury as dependent variables (except WHO ordinal scale for which linear regression was used) and HS as the main independent variable. The multivariable model was adjusted for age, sex, race, recent healthcare exposure (hospitalization or nursing facility residence $<90$ days before COVID-19 diagnosis), hypertension, and dyslipidemia.

All statistical analyses were performed using $\mathrm{R}$ version 3.6.1 (Vienna, Austria).

\section{Results}

The cohort included 342 patients, of whom 178 (52\%) had HS based on imaging or HSI (Fig. 1). Median follow-up was 113 days after COVID-19 diagnosis. Table 1 shows the characteristics of the cohort. Prevalence of metabolic comorbidities was high: $69 \%$ of patients had hypertension, $43 \%$ diabetes, $47 \%$ dyslipidemia, $85 \%$ overweight (BMI $>23$ for Asians or $>25$ for non-Asians), and $52 \%$ obesity. Patients with HS were younger and more often African-American compared to those without HS.

Overall, $19 \%$ of patients died and $>50 \%$ required ICU admission (Table 1). In unadjusted analyses, patients with HS more often required intubation, dialysis, or vasopressors and had greater odds of transaminitis, but were less likely to die (Tables 1, 2, Fig. 2). There was no difference by HS status in ICU admission or jaundice. In multivariable analyses, HS was associated with increased odds of ICU admission (odds ratio 1.60 [95\% confidence interval 1.00-2.57]), intubation (2.51 [1.52-4.16]), vasopressor requirement (1.22 [1.11-1.34]), and ALT > 5x ULN (2.12 [1.03-4.37]). Patients with HS had more severe disease based on higher WHO ordinal scale (Table 2). Rates of new/worsening ascites and encephalopathy were low: $1.3 \%$ and $2.5 \%$, respectively, with no difference based on HS status $(P>0.4)$.

We conducted sensitivity analyses where only patients with imaging evidence of hepatic steatosis $>30$ days prior to COVID-19 diagnosis. Of 141 patients with prior imaging, 68 (48\%) had HS. Imaging evidence of HS was associated with increased adjusted odds of intubation (2.75 [1.21-6.25]), vasopressor requirements (1.22 [1.04-1.43]), and odds of ALT $>5 x$ ULN (7.09 [1.73-28.95]) (Table 3). There was no difference by HS status in ICU admission, death, jaundice, dialysis requirement, or WHO ordinal scale.

\section{Discussion}

We found that in a US cohort of hospitalized patients with COVID-19, HS was associated with increased disease severity and transaminitis, but not with clinically relevant liver complications such as jaundice or new/worsening ascites or hepatic encephalopathy.

Our study adds to a literature on the effects of HS, and chronic liver disease more generally, on COVID-19 severity. Two studies found that HS based on imaging or HSI was associated with increased transaminitis and more severe respiratory disease $[11,15]$, while in a third it was 
Fig. 1 Study flowchart

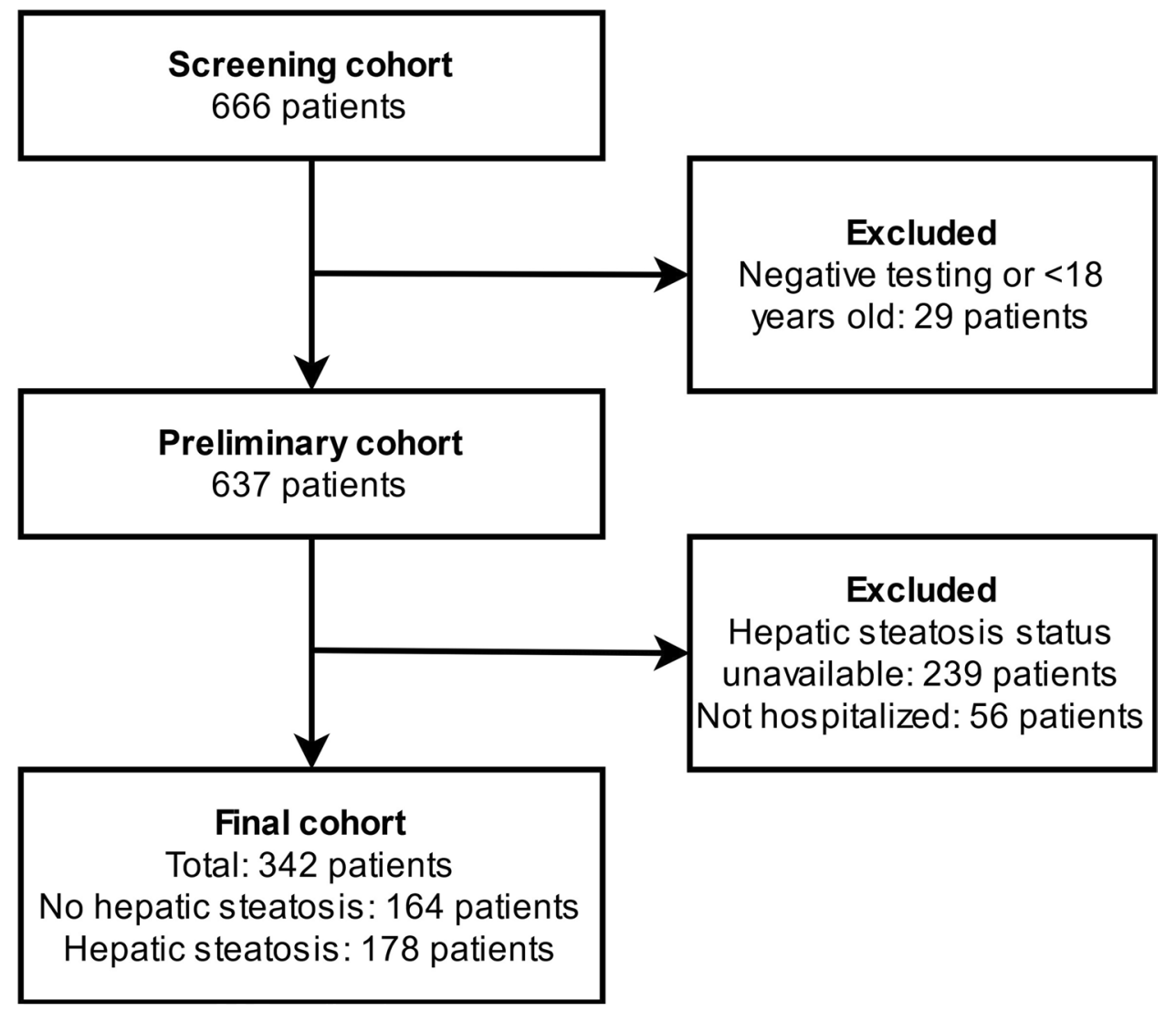

only patients with HS and advanced fibrosis/cirrhosis who suffered poorer outcomes, not those with HS without fibrosis [16]. Notably, all these studies were in cohorts from China, with far lower rates of underlying obesity, diabetes, and other comorbidities than in our cohort. In addition to HS, chronic liver disease generally has also been associated with increased disease severity in COVID-19 [4, 19]. We were unable to evaluate the effect of non-HS chronic liver disease on disease severity as prevalence was low in this cohort.

Transaminitis with ALT $>2 \mathrm{x}$ ULN was common in our cohort, and higher in individuals with HS even accounting for baseline ALT. However, severe liver complications such as jaundice, ascites, and encephalopathy were uncommon and unaffected by the presence of HS. This finding is consistent with other literature on high frequency of transaminitis in COVID-19-which is also associated with poorer outcomes severity-but low rates of jaundice [17, 18]. International studies have found that among patients with underlying chronic liver disease with or without cirrhosis, acute liver injury on presentation was $>30 \%[4,19]$. As our population had a low prevalence of cirrhosis ( $2 \%$ ), we lacked power to identify such a trend. However, the low rates of decompensation in a real-world cohort of hospitalized patients not otherwise enriched for liver disease, over half of whom required ICU admission, suggest that in COVID-19 liver disease likely reflects underlying sepsis and is not a primary driver of morbidity.

Limitations of this study include use of a surrogate measure of HS. We addressed this with a sensitivity analysis including only patients with prior imaging and found that HS prevalence based on imaging or HSI was not substantially higher than based on imaging alone. While prevalence of HS was higher than that of the general population in this cohort, this high prevalence likely reflects the metabolic comorbidities including diabetes and obesity among this study population. Strengths include that this is one of the first studies of its kind in the USA and we utilized robust classification through chart review.

In conclusion, the presence of HS is associated with more severe COVID-19 respiratory disease. Liver-related complications were low and had no association with HS. 
Table 1 Clinical characteristics based on hepatic steatosis status

\begin{tabular}{|c|c|c|c|c|}
\hline Characteristic & Overall $(N=342)$ & No hepatic steatosis $(n=164)$ & Hepatic steatosis $(n=178)$ & $P$ value \\
\hline \multicolumn{5}{|l|}{ Demographics } \\
\hline Age & $63.0(52.0-73.0)$ & $66.5(54.0-79.2)$ & $58.5(49.0-67.0)$ & $<0.001$ \\
\hline$\%$ male & $53.5 \%$ & $57.3 \%$ & $50.0 \%$ & 0.19 \\
\hline Race $(n=338)$ & & & & $<0.001$ \\
\hline White & $44.7 \%$ & $54.9 \%$ & $35.1 \%$ & \\
\hline Black & $44.4 \%$ & $35.4 \%$ & $52.9 \%$ & \\
\hline Other & $10.9 \%$ & $9.8 \%$ & $12.1 \%$ & \\
\hline \multicolumn{5}{|l|}{ Comorbidities } \\
\hline Body mass index $(n=337)$ & $30.0(25.9-36.0)$ & $26.6(24.8-29.2)$ & $34.7(30.3-40.7)$ & $<0.001$ \\
\hline Hypertension $(n=341)$ & $69.2 \%$ & $67.7 \%$ & $70.6 \%$ & 0.56 \\
\hline Dyslipidemia $(n=340)$ & $47.0 \%$ & $47.6 \%$ & $46.6 \%$ & 0.91 \\
\hline Diabetes & $43.3 \%$ & $37.8 \%$ & $48.3 \%$ & 0.06 \\
\hline \multicolumn{5}{|l|}{ Baseline laboratory values } \\
\hline Creatinine $(\mathrm{mg} / \mathrm{dL})(n=325)$ & $1.0(0.8-1.3)$ & $1.0(0.8-1.5)$ & $1.0(0.8-1.2)$ & 0.11 \\
\hline $\operatorname{ALT}(\mathrm{U} / \mathrm{L})(n=326)$ & $21.0(15.0-30.0)$ & $19.0(14.0-28.0)$ & $23.0(16.5-33.0)$ & $<0.001$ \\
\hline Total bilirubin $(\mathrm{mg} / \mathrm{dL})(n=322)$ & $0.5(0.4-0.7)$ & $0.5(0.4-0.7)$ & $0.5(0.4-0.7)$ & 0.80 \\
\hline Hemoglobin A1c (\%) $(n=242)$ & $6.2(5.7-7.3)$ & $6.0(5.6-6.8)$ & $6.3(5.8-7.6)$ & 0.01 \\
\hline High-density lipoprotein $(\mathrm{mg} / \mathrm{dL})(n=251)$ & $46.0(38.0-56.0)$ & $46.0(37.0-56.0)$ & $45.0(38.2-55.8)$ & 0.70 \\
\hline Low-density lipoprotein $(\mathrm{mg} / \mathrm{dL})(n=247)$ & $91.0(68.0-120.5)$ & $88.0(64.0-114.0)$ & $92.5(72.0-125.0)$ & 0.11 \\
\hline Triglycerides $(\mathrm{mg} / \mathrm{dL})(n=251)$ & $124.0(85.5-178.0)$ & $121.5(86.2-172.2)$ & $125.0(85.0-184.0)$ & 0.79 \\
\hline \multicolumn{5}{|l|}{ Peak laboratory values } \\
\hline Creatinine $(\mathrm{mg} / \mathrm{dL})(n=340)$ & $1.3(0.9-2.9)$ & $1.3(0.9-2.7)$ & $1.2(0.9-3.2)$ & 0.85 \\
\hline Alanine aminotransferase (U/L) $(n=331)$ & $50.0(31.0-96.5)$ & $41.0(26.2-75.0)$ & $59.0(36.0-114.0)$ & $<0.001$ \\
\hline Total bilirubin $(\mathrm{mg} / \mathrm{dL})(n=331)$ & $0.7(0.5-1.0)$ & $0.6(0.4-1.0)$ & $0.8(0.5-1.0)$ & 0.02 \\
\hline \multicolumn{5}{|l|}{ Disease severity } \\
\hline Death & $18.7 \%$ & $22.6 \%$ & $15.2 \%$ & 0.01 \\
\hline Intensive care unit & $54.1 \%$ & $51.2 \%$ & $56.7 \%$ & 0.33 \\
\hline Intubation & $34.5 \%$ & $25.0 \%$ & $43.3 \%$ & $<0.001$ \\
\hline Dialysis & $12.0 \%$ & $8.0 \%$ & $15.7 \%$ & 0.03 \\
\hline Vasopressors & $24.0 \%$ & $14.7 \%$ & $32.6 \%$ & $<0.001$ \\
\hline ALT $>2 x$ ULN & $45.3 \%$ & $39.2 \%$ & $50.9 \%$ & 0.04 \\
\hline ALT $>5 x$ ULN & $13.6 \%$ & $8.2 \%$ & $18.5 \%$ & 0.007 \\
\hline Bilirubin $>2 \mathrm{mg} / \mathrm{dL}$ & $8.8 \%$ & $8.9 \%$ & $8.7 \%$ & 1 \\
\hline Bilirubin $>4 \mathrm{mg} / \mathrm{dL}$ & $2.1 \%$ & $3.2 \%$ & $1.2 \%$ & 0.27 \\
\hline World Health Organization ordinal scale & $4.0(4.0-7.0)$ & $4.0(3.0-7.0)$ & $5.0(4.0-7.0)$ & 0.34 \\
\hline \multicolumn{5}{|l|}{ Treatment } \\
\hline Any treatment & $57.9 \%$ & $54.3 \%$ & $61.2 \%$ & 0.23 \\
\hline Hydroxychloroquine & $34.8 \%$ & $32.9 \%$ & $36.6 \%$ & 0.49 \\
\hline Remdesivir & $5.2 \%$ & $5.7 \%$ & $4.7 \%$ & 0.80 \\
\hline Tocilizumab & $16.7 \%$ & $10.8 \%$ & $22.1 \%$ & 0.01 \\
\hline Corticosteroids & $13.6 \%$ & $10.8 \%$ & $16.3 \%$ & 0.15 \\
\hline Sarilumab (blinded trial) & $5.0 \%$ & $4.9 \%$ & $5.1 \%$ & 1.00 \\
\hline Donor plasma & $0.6 \%$ & $0.6 \%$ & $0.6 \%$ & 1.00 \\
\hline
\end{tabular}

$A L T$ alanine aminotransferase, $U L N$ upper limit of normal, defined as the greater of the patient's prior baseline (if known), $19 \mathrm{U} / \mathrm{L}$ for women, or $30 \mathrm{U} / \mathrm{L}$ for men 
Table 2 Predictors of disease severity and liver injury based on hepatic steatosis as defined by hepatic steatosis index and imaging

Fig. 2 Frequency of coronavirus disease-2019 severity and liver injury based on hepatic steatosis status

\begin{tabular}{|c|c|c|c|c|}
\hline & Univariable & & Multivariable $^{\mathrm{a}}$ & \\
\hline \multicolumn{5}{|l|}{ Logistic regressions } \\
\hline Outcome & Odds ratio & $P$ value & Odds ratio & $P$ value \\
\hline Death & $0.61(0.35,1.06)$ & 0.08 & $0.94(0.49,1.78)$ & 0.84 \\
\hline Intensive care unit admission & $1.25(0.82,1.91)$ & 0.31 & $1.60(1.00,2.57)$ & 0.05 \\
\hline Intubation & $2.29(1.44,3.63)$ & $<0.001$ & $2.51(1.52,4.16)$ & $<0.001$ \\
\hline Dialysis & $2.15(1.07,4.32)$ & 0.03 & $1.72(0.81,3.65)$ & 0.16 \\
\hline Vasopressor use & $2.80(1.64,4.78)$ & $<0.001$ & $1.22(1.11,1.34)$ & $<0.001$ \\
\hline ALT $>2 x$ ULN & $1.60(1.04,2.48)$ & 0.03 & $1.44(0.90,2.32)$ & 0.13 \\
\hline ALT $>5 x$ ULN & $2.53(1.28,5.02)$ & 0.01 & $2.12(1.03,4.37)$ & 0.04 \\
\hline Bilirubin $>2 \mathrm{mg} / \mathrm{dL}$ & $0.98(0.46,2.09)$ & 0.95 & $0.86(0.38,1.95)$ & 0.72 \\
\hline Bilirubin $>4 \mathrm{mg} / \mathrm{dL}$ & $0.36(0.07,1.87)$ & 0.22 & $0.39(0.07,2.30)$ & 0.30 \\
\hline \multicolumn{5}{|l|}{ Linear regression } \\
\hline Outcome & Beta & $P$ value & Beta & $P$ value \\
\hline WHO ordinal scale & $0.21(-0.19,0.61)$ & 0.30 & $0.45(0.03,0.86)$ & 0.04 \\
\hline
\end{tabular}

Bold values indicate statistically-significant association

$A L T$ alanine aminotransferase, $I C U$ intensive care unit, $U L N$ upper limit of normal, defined as the greater of the patient's prior baseline (if known), $19 \mathrm{U} / \mathrm{L}$ for women, or $30 \mathrm{U} / \mathrm{L}$ for men, WHO World Health Organization

${ }^{a}$ Adjusted for age, sex, race (white, black, or other), recent healthcare exposure (hospitalization or residence at an extended care facility or skilled nursing facility $<90$ days before COVID-19 diagnosis), presence of hypertension, and presence of dyslipidemia

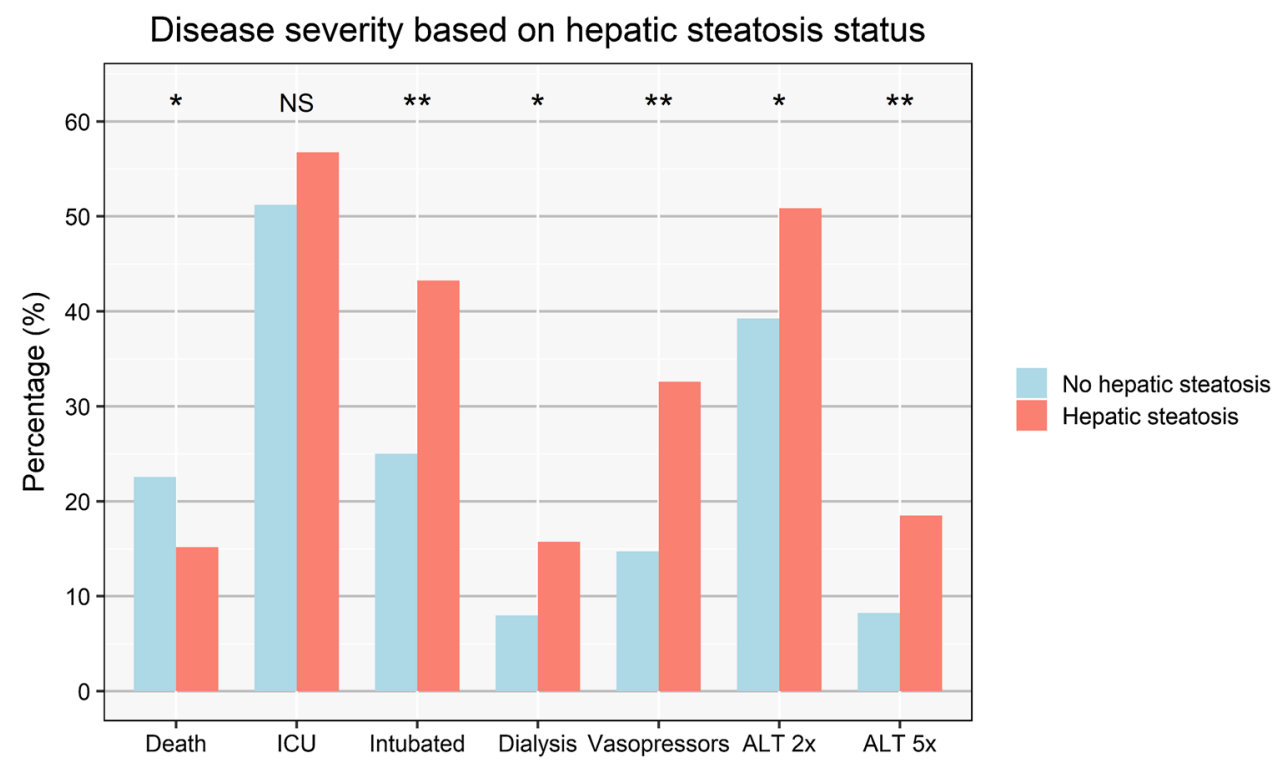


Table 3 Predictors of disease severity and liver injury based on hepatic steatosis as defined by imaging alone

\begin{tabular}{|c|c|c|c|c|}
\hline & Univariable & & Multivariable $^{\mathrm{a}}$ & \\
\hline \multicolumn{5}{|l|}{ Logistic regressions } \\
\hline Outcome & Odds ratio & $P$ value & Odds ratio & $P$ value \\
\hline Death & $0.65(0.29,1.49)$ & 0.31 & $0.83(0.31,2.20)$ & 0.71 \\
\hline Intensive care unit admission & $1.26(0.64,2.47)$ & 0.50 & $1.68(0.76,3.71)$ & 0.20 \\
\hline Intubation & $2.53(1.25,5.12)$ & 0.01 & $2.75(1.21,6.25)$ & 0.02 \\
\hline Dialysis & $1.54(0.58,4.11)$ & 0.38 & $1.89(0.61,5.86)$ & 0.27 \\
\hline Vasopressor use & $2.83(1.25,6.40)$ & 0.01 & $1.22(1.04,1.43)$ & 0.02 \\
\hline ALT $>2 x$ ULN & $2.28(1.14,4.55)$ & 0.02 & $3.36(1.43,7.87)$ & 0.01 \\
\hline ALT $>5 x$ ULN & $7.11(1.97,25.71)$ & 0.003 & $7.09(1.73,28.95)$ & 0.01 \\
\hline Bilirubin $>2 \mathrm{mg} / \mathrm{dL}$ & $1.79(0.55,5.77)$ & 0.33 & $2.40(0.61,9.49)$ & 0.21 \\
\hline Bilirubin $>4 \mathrm{mg} / \mathrm{dL}$ & $0.52(0.05,5.90)$ & 0.60 & $3.63(0.12,109.38)$ & 0.46 \\
\hline \multicolumn{5}{|l|}{ Linear regression } \\
\hline Outcome & Beta & $P$ value & Beta & $P$ value \\
\hline WHO ordinal scale & $0.28(-0.35,0.91)$ & 0.38 & $0.49(-0.23,1.21)$ & 0.18 \\
\hline
\end{tabular}

Bold values indicate statistically-significant association

$A L T$ alanine aminotransferase, $I C U$ intensive care unit, $U L N$ upper limit of normal, defined as the greater of the patient's prior baseline (if known), $19 \mathrm{U} / \mathrm{L}$ for women, or $30 \mathrm{U} / \mathrm{L}$ for men, $W H O$ World Health Organization

${ }^{a}$ Adjusted for age, sex, race (white, black, or other), recent healthcare exposure (hospitalization or residence at an extended care facility or skilled nursing facility $<90$ days before COVID-19 diagnosis), presence of hypertension, and presence of dyslipidemia
Author's contributions Dr. PS and VC are the guarantors of the article and developed the concept, contributed to study design, data analysis, and interpretation, and drafted the manuscript. FH contributed to study design and data analysis and critically reviewed the manuscript. JB contributed to study design, collected the data, and critically reviewed the manuscript. CR, IK, KP, C-YH, CS, and JL collected the data and critically reviewed the manuscript. NG and PS developed the concept, contributed to study design, data analysis, and interpretation, and critically reviewed the manuscript. All authors identified above have critically reviewed the paper and approve the final version of this paper, including the authorship statement.

Funding VLC was supported by an AASLD Clinical, Translational and Outcomes Research Award. JL was supported by an NIH Training Grant in Epidemiology and Health Services (T32DK062708).

\section{Compliance with Ethical Standards}

Conflict of interest Vincent Chen, Fadi Hawa, Jeffrey Berinstein, Chanakyaram Reddy, Ihab Kassab, Kevin Platt, Chia-Yang Hsu, Jeremy Louissaint, Calen Steiner, and Pratima Sharma: no financial conflicts of interest to disclose. Naresh Gunaratnam: Co-founder and CMO, Lean Medical, LL. Speaker, Nestle Health Services.

\section{References}

1. Dong E, Du H, Gardner L. An interactive web-based dashboard to track COVID-19 in real time. Lancet Infect Dis. 2020;20:533-534.

2. Pan L, Mu M, Yang P, et al. Clinical characteristics of COVID19 patients with digestive symptoms in Hubei, China: a descriptive, cross-sectional, multicenter study. Am J Gastroenterol. 2020;115:766-773.

3. Zhang C, Shi L, Wang F-S. Liver injury in COVID-19: management and challenges. Lancet Gastroenterol Hepatol. 2020;5:428-430.

4. Moon AM, Webb GJ, Aloman C, et al. High mortality rates for SARS-CoV-2 infection in patients with pre-existing chronic liver disease and cirrhosis: preliminary results from an international registry. J Hepatol. 2020. https://doi.org/10.1016/j. jhep.2020.05.013

5. Le MH, Devaki P, Ha NB, et al. Prevalence of non-alcoholic fatty liver disease and risk factors for advanced fibrosis and mortality in the United States. PLoS ONE. 2017;12:e0173499.

6. Eguchi $\mathrm{Y}$, Hyogo $\mathrm{H}$, Ono M, et al. Prevalence and associated metabolic factors of nonalcoholic fatty liver disease in the general population from 2009 to 2010 in Japan: a multicenter large retrospective study. J Gastroenterol. 2012;47:586-595.

7. Lazo M, Hernaez R, Eberhardt MS, et al. Prevalence of nonalcoholic fatty liver disease in the United States: the Third National Health and Nutrition Examination Survey, 1988-1994. Am J Epidemiol. 2013;178:38-45.

8. Williams CD, Stengel J, Asike MI, et al. Prevalence of nonalcoholic fatty liver disease and nonalcoholic steatohepatitis among a largely middle-aged population utilizing ultrasound and liver biopsy: a prospective study. Gastroenterology. 2011;140:124-131.

9. Guo W, Li M, Dong Y, et al. Diabetes is a risk factor for the progression and prognosis of COVID-19. Diabetes Metab Res Rev. 2020. https://doi.org/10.1002/dmrr.3319.

10. Qingxian C, Fengjuan C, Fang L, et al. Obesity and COVID-19 severity in a designated hospital in Shenzhen, China. Lancet Infect Dis. 2020;43:1392-1398.

11. Ji D, Qin E, Xu J, et al. Non-alcoholic fatty liver diseases in patients with COVID-19: a retrospective study. J Hepatol. 2020;1:3. 
12. Kwo PY, Cohen SM, Lim JK. ACG clinical guideline: evaluation of abnormal liver chemistries. Am J Gastroenterol. 2017;112:18-35.

13. Terrault NA, Bzowej NH, Chang KM, et al. AASLD guidelines for treatment of chronic hepatitis B. Hepatology. 2016;63:261-283.

14. Lee JH, Kim D, Kim HJ, et al. Hepatic steatosis index: a simple screening tool reflecting nonalcoholic fatty liver disease. Dig Liver Dis. 2010;42:503-508.

15. Gao F, Zheng KI, Wang XB, et al. Metabolic associated fatty liver disease increases COVID-19 disease severity in non-diabetic patients. J Gastroenterol Hepatol. 2020. https://doi.org/10.1111/ jgh.15112.

16. Targher G, Mantovani A, Byrne CD, et al. Risk of severe illness from COVID-19 in patients with metabolic dysfunction-associated fatty liver disease and increased fibrosis scores. Gut. 2020. https://doi.org/10.1136/gutjnl-2020-321611.

17. Bangash MN, Patel J, Parekh D. COVID-19 and the liver: little cause for concern. Lancet Gastroenterol Hepatol. 2020;5:529-530.
18. Kulkarni AV, Kumar P, Tevethia HV, et al. Systematic review with meta-analysis: liver manifestations and outcomes in COVID-19. Aliment Pharmacol Ther. 2020;52:584-599.

19. Sarin SK, Choudhury A, Lau GK, et al. Pre-existing liver disease is associated with poor outcome in patients with SARS CoV2 infection; The APCOLIS Study (APASL COVID-19 Liver Injury Spectrum Study). Hepatol Int. 2020. https://doi.org/10.1007/ s12072-020-10072-8.

Publisher's Note Springer Nature remains neutral with regard to jurisdictional claims in published maps and institutional affiliations. 